\title{
Gadolinium-based Contrast Agent P03277
}

National Cancer Institute

\section{Source}

National Cancer Institute. Gadolinium-based Contrast Agent P03277. NCI Thesaurus.

Code C128640.

A gadolinium-based paramagnetic contrast agent, with potential imaging enhancing activity upon magnetic resonance imaging (MRI). Upon administration of P03277 and placement in a magnetic field, this agent produces a large magnetic moment and creates a large local magnetic field, which can enhance the relaxation rate of nearby protons. This change in proton relaxation dynamics, increases the MRI signal intensity of tissues in which this agent has accumulated; therefore, contrast and visualization of those tissues is enhanced compared to unenhanced MRI. 